\title{
The role of the ratio of vector and host densities in the evolution of transmission modes in vector-borne diseases. The example of sylvatic Trypanosoma cruzi
}

\author{
Perrine Pelosse $^{a}$, Christopher M. Kribs-Zaleta ${ }^{a, *}$ \\ ${ }^{a}$ Mathematics Department, University of Texas at Arlington \\ Box 19408, Arlington, TX 76019-0408 USA
}

June 25, 2012

\begin{abstract}
Pathogens may use different routes of transmission to maximize their spread among host populations. Theoretical and empirical work conducted on directly-transmitted diseases suggest that horizontal (i.e., through host contacts) and vertical (i.e., from mother to offspring) transmission modes trade off, on the ground that highly virulent pathogens, which produce larger parasite loads, are more efficiently transmitted horizontally, and that less virulent pathogens, which impair host fitness less significantly, are better transmitted vertically. Other factors than virulence such as host density could also select for different transmission modes, but they have barely been studied. In vector-borne diseases, pathogen transmission rate is strongly affected by host-vector relative densities and by processes of saturation in contacts between hosts and vectors. The parasite Trypanosoma cruzi which is transmitted by triatomine bugs to several vertebrate hosts is responsible for Chagas' disease in Latin America. It is also widespread in sylvatic cycles in the southeastern U.S. in which it typically induces no mortality costs to its customary hosts. Besides classical transmission via vector bites, alternative ways to generate infections in hosts such as vertical and oral transmission (via the consumption of vectors by hosts) have been reported in these cycles. The two major T. cruzi strains occurring in the U.S. seem to exhibit differential efficiencies at vertical and classical horizontal transmissions. We investigated whether the vector-host ratio affects the outcome of the competition between the two parasite strains using an epidemiological two-strain model considering all possible transmission routes for sylvatic $T$. cruzi. We were able to show that the vector-host ratio influences the evolution of transmission modes providing that oral transmission is included in the model as a possible transmission mode, that oral and classical transmissions saturate at different vector-host ratios and that the vector-host ratio is between the two saturation thresholds. Even if data on parasite strategies and demography of hosts and vectors in the field are crucially lacking to test to what extent the conditions needed for the vector-host ratio to influence evolution of transmission modes are plausible, our results open new perspectives for understanding the specialization of the two major T. cruzi strains occurring in the U.S. Our work also provides an original theoretical framework to investigate the evolution of alternative transmission modes in vector-borne diseases.
\end{abstract}

Keywords: vector-borne diseases, trade-off, vertical transmission, horizontal transmission, oral transmission, stercorarian transmission, contact saturation process, host density

* Corresponding author: kribs@uta.edu, tel. +1 817272 5513, fax +1 8172725802 


\section{Introduction}

Pathogens may use different routes of infection in order to maximize their transmission in host populations. Intrinsic characteristics of hosts and pathogens as well as the biodemography of host populations may favor one transmission route over another. Theoretical (e.g. [1]) and empirical studies (e.g. [2, 3, 4]) suggest that pathogen virulence is responsible for a trade-off between horizontal (i.e., via contacts between infected and uninfected hosts) and vertical (i.e., from infected mother to offspring) transmission modes. Indeed, increased levels of virulence would translate into high parasite loads in the organism (i.e., parasitemia) and thus favor pathogen horizontal transmission, but impair host fitness and consequently the likeliness of vertical transmission [1]. Yet, other determinants than virulence could lead pathogens to specialize for different modes of transmission. Host density for instance, acting together with virulence, has been hypothesized to affect the success of horizontal versus vertical transmission: a highly virulent parasite resorting to horizontal transmission and causing too much harm to its hosts is expected to be counter-selected when host density is low [2]. Host density independently of virulence could also select for different modes of transmissions, in particular relative host densities in the case of multihost parasites, which have been shown to select for switches from simple to complex parasite life cycles [5].

Vector-borne diseases, i.e., diseases transmitted between hosts by blood-sucking arthropods, are a major health threat in tropical and subtropical regions and put temperate regions at risk because of climate change. While alternative transmission routes such as vertical transmission have been reported among vector-borne diseases (e.g., malaria [6], Chagas' disease [7]) their selective advantages over classical means of infection have been largely overlooked. In vector-borne diseases, not only the densities of hosts and vectors, but also the ratio of their densities, have strong implications for pathogen transmission $[8,9,10]$. The per-vector biting rate typically increases as the number of hosts available per vector increases, and saturates at the vectors' desired feeding rate for high host-vector ratios. As a consequence, the total contact rate between hosts and vectors depends on hosts for relatively low host-vector ratios and on vectors for relatively high host-vector ratios (see [10]). Saturation processes have been used extensively in ecology to model interacting populations (e.g., predator-prey systems) but quite surprisingly, they have not been applied in epidemiology to the modeling of vectorborne diseases (but see $[10,11,12,13]$ ). Instead, epidemiological studies modeling host and vector populations traditionally follow the assumption developed for malaria by Ross [8] that hosts are numerous and do not constitute a limited resource for vectors. In such models, the per-vector biting rate is always maximal and independent of the host-vector ratio, and the total contact rate only limited by vector density. Chagas' disease vectors, the Triatomines (see below), are however known to induce irritability in their hosts when the number of their bites is too high [14], which is likely to be the case among other vector-borne diseases.

The protozoan parasite Trypanosoma cruzi infects over 100 mammalian host species in the Americas and is responsible for Chagas' disease in Latin America. T. cruzi is transmitted between hosts by blood-sucking insects of the subfamily Triatominae. In the southeastern U.S. (SE US), the two most widespread strains, T. cruzi I and IIa (recently renamed IV) [15], use as primary hosts wild mammals such as opossums, raccoons or woodrats. SE US T. cruzi strains occur almost exclusively in sylvatic cycles and typically induce only mild 
symptoms on their hosts $[16,17]$. Cross-immunity is known to occur between T. cruzi strains (e.g. [18]), i.e., infection of a host by one strain induces immunity to future infections by other strains. Mixed infections can however occur among vectors [19]. The classical infection route for T. cruzi involves a healthy vector feeding on an infected host, referred to as bloodmeal transmission, and an infected vector feeding on a healthy host. For the latter transmission to be efficient, defecation has to occur shortly after the bite and the parasites present in the faeces have to reach host blood via either mucous membranes or skin lesions surrounding the bite area. Stercorarian transmission is therefore quite a hazardous route of transmission compared to transmission through the salivary glands that takes place in other vector-borne diseases such as malaria. In addition, in the SE US, the two main vectors involved in T. cruzi transmission, Triatoma sanguisuga and Triatoma gerstaeckeri [20], appear to display cautious behaviors when they feed on their hosts (in particular they avoid climbing completely onto the host) and a longer delay between feeding and defecating compared to South American species (e.g., [21]).

Stercorarian transmission added to the supposed inefficiency of SE US vectors to pass the infection to hosts may have led $T$. cruzi to favor or adapt to alternative transmission routes $[11,13]$. Vertical transmission has been reported among placental hosts such as rats and mice [22, 23], and humans [24]. In addition, sylvatic hosts such as raccoons and opossums are opportunistic feeders whose diet includes insects, and experimental studies have shown that they can get infected by ingesting infected vectors $[25,26]$. Oral transmission of $T$. cruzi is likely to be a dominant transmission pathway for sylvatic T. cruzi in the SE US, and explain the high disease prevalence observed among raccoons in particular [27], as suggested by the model of [13]. Vertical and oral transmissions therefore provide two alternative ways to generate new infections among hosts and might serve as alternatives to the classical stercorarian transmission route.

Interestingly, even if pathogen traits such as parasitemia, infectivity and transmissibility seem highly correlated, beyond pathogen genotype, to the geographical origin of the pathogen strain and the host considered (e.g., see contradictory results of [28] and [27]), the two T. cruzi strains occurring in the SE US, types I and IIa, seem to differ regarding their classical (i.e., stercorarian) and vertical transmission efficiencies. A comparison of the epidemiological behaviours of type I and IIa Brazilian strains inside vectors demonstrated that T. cruzi I develops larger parasitemias and performs better in mixed infection than T. cruzi IIa [29]. It is therefore most likely that type I overcomes type II at stercorarian transmission efficiency. In regard to vertical transmission, an experiment showed that mice infected experimentally with T. cruzi IIa transmit the parasite to 66 percent of their offspring, compared to 33 percent for $T$. cruzi I, although type IIa parasitemias are drastically smaller than type I ones [23]. T. cruzi IIa seems therefore better adapted to vertical transmission, possibly via a better ability to invade reproductive tissues compared to T. cruzi I $[23,30]$.

The present study uses a theoretical modeling framework to address the question of whether different modes of transmission can be selected in response to the population dynamics of hosts and vectors, and in particular, to the vector-host density ratio, which has a strong impact on the transmission rate of the disease. Trypanosoma cruzi can infect different host and vector species, and vector-host ratios can vary considerably among the host-vector 
associations considered, as shown by the estimations of [10]. Because parasite-induced mortality has never been reported among customary sylvatic SE US hosts, we will not consider in our model that hosts are submitted to an additive mortality rate when infected, contrary to classical models developed on the evolution of transmission modes. This will allow us to test whether host and vector densities alone, and in particular whether the ratio of their densities, could drive a specialization toward one transmission mode or another. Demonstrating that the relative densities of vectors and hosts are a significant selective force driving the divergence of $T$. cruzi strains could bring new elements into the understanding of the epidemiology of this disease in the U.S., but also in Latin America, given the variability of potential vector and host species and climates and habitats. More broadly, this study also brings new elements into the comprehension of the evolution of the trade-off between horizontal and vertical transmission in a vector-borne transmission setting, which has never been done before.

\section{Model}

\subsection{Saturation in infection rates}

Since contact processes drive infection dynamics and are especially complex for vector-borne infections, we begin by describing how contacts between two distinct populations are modeled, before explaining how this leads to saturation in T. cruzi infection rates in particular. Contacts between separate populations, such as hosts and vectors, predators and prey, or individuals of different risk levels, can be considered to be initiated by one of the populations, and the rate at which such contacts occur can be described in terms of a single member of this population. The initiating individual generally has a preferred contact frequency, say $C$, with the target population. The actual contact rate, however, is also typically a function of the ratio of the densities of the two populations $[8,31]$, say $Q$. In the case of vector bloodmeals, these are the vectors' desired feeding frequency and the host/vector ratio. In the case of predation, the predator's functional response increases as a function of the prey-predator ratio and saturates for high prey-predator ratios since predators can then feed at their preferred rate. When the target population is relatively scarce $(Q$ small), contacts will be more difficult, so the actual per capita contact rate will be lower than $C$; when it is relatively plentiful ( $Q$ large), the contact process will be saturated in the target population, and the per capita contact rate will approach (or reach) $C$. The simplest way to model this saturation is with a Holling type I rate $[12,32], c(Q)=C \min \left(Q / Q_{*}, 1\right)$, in which the contact rate per initiator increases linearly in $Q$ up to some threshold value $Q_{*}$ at which it reaches the maximum rate $C$ (other ways to model this saturation, such as Holling type II and III functions, use a more gradual curve). The total contact rate is then given by multiplying the per capita rate $c$ by the initiating population density, which makes the product linear in the initiating (vector or predator) density if $Q>Q_{*}$ (targets are plentiful) but linear in the target population density if $Q<Q_{*}$ (targets are scarce).

Our model encompasses all the different transmission routes reported for T. cruzi, all but one of which (vertical transmission in hosts) involve a contact process. The transmission routes that involve contacts between hosts and vectors are transmission by predation of hosts on vectors (oral transmission), and transmission by bloodmeal of vectors on hosts, the latter potentially leading to infection in vectors (bloodmeal transmission) or infection in hosts 


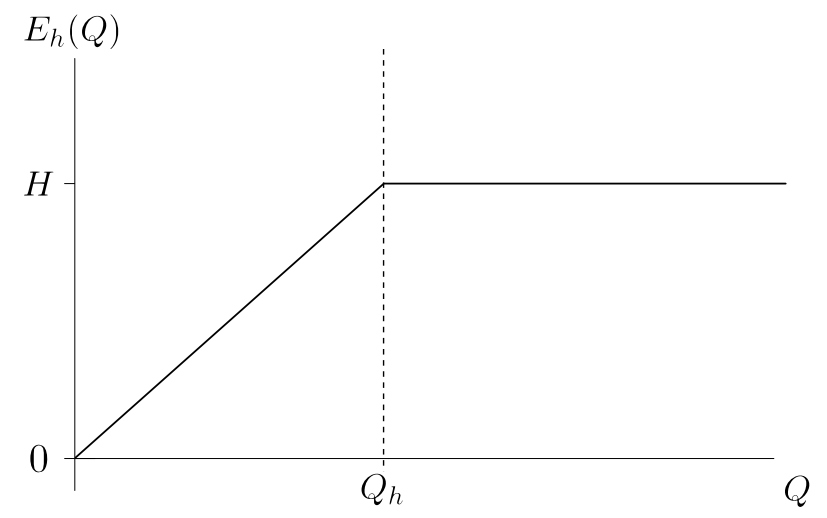

Figure 1: Predation effort $E_{h}$ as a function of $Q . E_{h}$ is an increasing function of $Q$ for $Q<Q_{h}$ and then saturates for $Q>Q_{h}$.

(stercorarian transmission). Mixing of vectors and hosts is assumed to be homogeneous. In modeling them, we allow parasite strains $i(i=1,2)$ ( 1 and 2 being implicitly used thereafter to refer to $T$. cruzi strains I and IIa, respectively) to differ in their efficiencies to be transmitted via these different pathways.

In sylvatic T. cruzi systems, oral transmission occurs via host predation on vectors. We describe this saturation process using Holling type I saturation. Then, the predator functional response $E_{h}$ (in vectors/host/time), i.e., the per predator (here host) contact rate, increases linearly in the vector-host ratio until it reaches the threshold:

$$
E_{h}(Q)=H \min \left(Q / Q_{h}, 1\right),
$$

where $Q=N_{v} / N_{h}$ is the vector-host population density ratio [31], and $Q_{h}$ the threshold vector-host ratio above which contact rate saturates, i.e., vectors are plentiful and hosts can eat them at their preferred rate $H$ (in vector/host/time) (see Figure 1). By $N_{v}$ we denote only those vectors associated with the given host (vector density estimates in [12] used to derive raccoon-related vector/host ratio estimates discussed in this study have been scaled down to apportion vectors between their primary hosts, raccoons and opossums). We then assume that a proportion $\rho_{i}$ of hosts that consume a vector infected with strain $i$ will become infected.

For vectors taking their bloodmeals on hosts, contact rates can be modelled the same way. Whereas classical models of the transmission of vector-borne diseases typically assume that hosts are non-limiting, thus making the models valid only for low vector-host ratios [8, 9], we consider here that both vector and host densities vary in a way that either vectors or hosts can potentially be limiting. The per-vector biting rate is assumed to increase as the number of hosts per vector $1 / Q=N_{h} / N_{v}$ increases and to reach a plateau for high host-vector ratios. If, similarly, we use a Holling Type I saturation function, the per-vector biting rate (in contacts/vector/time) can be expressed as:

$$
b_{v} \min \left(\frac{1}{Q} / \frac{1}{Q_{v}}, 1\right)=b_{v} \min \left(Q_{v} / Q, 1\right),
$$

where $b_{v}$ is the vector preferred (maximal) biting rate (in contacts/vector/time) and $1 / Q_{v}$ the threshold host-vector ratio above which the contact rate saturates $[12,32]$. When hosts 


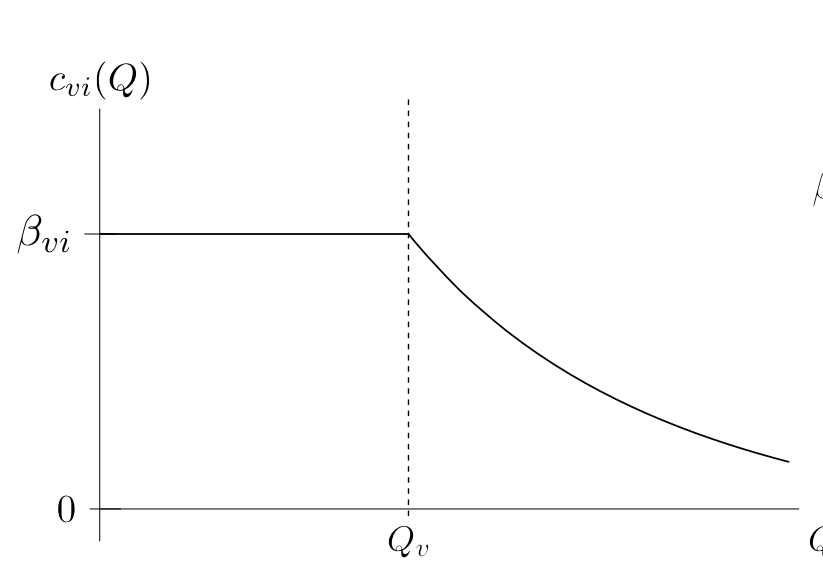

Figure 2: Infection rate of vectors $c_{v i}$ as a function of $Q . c_{v i}$ (in contacts/vector/time) saturates for $Q<Q_{v}$ and is then a decreasing function of $Q$ for $Q>Q_{v}$.

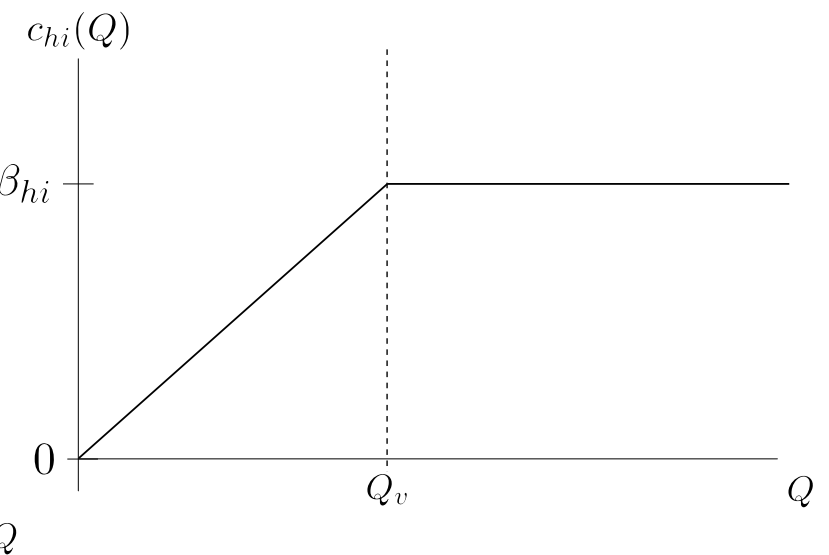

Figure 3: Stercorarian infection rate of hosts $c_{h i}$ as a function of $Q . c_{h i}$ (in contacts/host/time) is an increasing function of $Q$ for $Q<Q_{v}$ and then saturates for $Q>Q_{v}$.

are numerous relative to vectors (small $Q$ values), vectors are limiting and they can feed at their preferred rate $b_{v}$. The total number of contacts per time unit is therefore $b_{v} N_{v}$. When vectors are numerous relative to hosts (large $Q$ values), we consider that hosts tolerate bites at a maximum rate $b_{h}$ (in contacts/host/time). Since in that case, hosts are limiting, the total number of contacts per time unit is given by $b_{h} N_{h}$. $Q_{v}$ is therefore the threshold vector-host ratio where $b_{v} N_{v}=b_{h} N_{h}$, and is defined as $Q_{v}=b_{h} / b_{v}$ [10]. The infection rate of vectors (in $1 /$ time) is obtained by multiplying the per-vector biting rate by the probability $\pi_{v i}$ (in infected vectors per contact) that a contact between a host infected with strain $i$ and a susceptible vector gives rise to an infection in the vector:

$$
c_{v i}(Q)=b_{v} \min \left(Q_{v} / Q, 1\right) \pi_{v i}=\beta_{v i} \min \left(Q_{v} / Q, 1\right)
$$

in which $\beta_{v i}$, the (maximal) vector infection rate (in $1 /$ time), encapsulates both vector biting rate $b_{v}$ and probability of infection $\pi_{v i}$ (see Figure 2).

The infection rate of hosts via stercorarian transmission route, meanwhile, is calculated from this same contact process as the rate at which each host receives bites (the per-vector biting rate multiplied by the total number of vectors $N_{v}$ and divided by the total number of hosts $N_{h}$ ) multiplied by the probability $\pi_{h i}$ (in infected hosts per contact) that a contact between a vector infected by strain $i$ and a susceptible host leads to an infection in the host:

$$
c_{h i}(Q)=b_{v} \min \left(Q_{v} / Q, 1\right) \frac{N_{v}}{N_{h}} \pi_{h i}=\frac{b_{h}}{Q_{v}} \min \left(Q_{v} / Q, 1\right) Q \pi_{h i}=\beta_{h i} \min \left(Q / Q_{v}, 1\right) .
$$

Again, we define the (maximal) host infection rate $\beta_{h i}=b_{h} \pi_{h i}$ (in $1 /$ time) to simplify notation (see Figure 3).

From the preceding, one can see that transmission via bloodmeal (vector infection) saturates when vector-host ratios $Q$ are low, that is, when hosts are relatively abundant compared to vectors, which allows vectors to feed at their preferred rate. On the contrary, host infection via stercorarian and oral transmissions saturates when vectors are relatively abundant for 
Table 1: Variables and parameters used in the model

\begin{tabular}{cll}
\hline Var. & Definition & Units \\
\hline$S_{h}(t)$ & density of uninfected hosts & hosts/area \\
$I_{h i}(t)$ & density of hosts infected by strain $i$ & hosts/area \\
$S_{v}(t)$ & density of uninfected vectors & vectors/area \\
$I_{v i}(t)$ & density of vectors infected by strain $i$ & vectors/area \\
$Q(t)$ & vector-host population density ratio $\left(N_{v} / N_{h}\right)$ & vectors/host \\
$c_{h i}(Q(t))$ & stercorarian infection rate of hosts by strain $i$ & $1 /$ time \\
$c_{v i}(Q(t))$ & infection rate of vectors by strain $i$ & $1 /$ time \\
$E_{h}(Q(t))$ & per-host predation rate & vectors/host/time \\
$g_{h}$ & total host growth rate & $1 /$ time \\
$g_{v}$ & total vector growth rate & $1 /$ time \\
$r_{h}$ & maximum host growth rate & $1 /$ time \\
$r_{v}$ & maximum vector growth rate & $1 /$ time \\
$\mu_{h}$ & natural mortality rate of hosts & $1 /$ time \\
$\mu_{v}$ & natural mortality rate of vectors & $1 /$ time \\
$K_{h}$ & carrying capacity for hosts & hosts/area \\
$K_{v}$ & carrying capacity for vectors & vectors/area \\
$Q_{h}$ & threshold vector-host density ratio for predation & vectors/host \\
$Q_{v}$ & threshold vector-host density ratio for bloodmeals & vectors/host \\
$\beta_{h i}$ & stercorarian infection rate by strain $i$ & $1 /$ time \\
$\beta_{v i}$ & vector infection rate by strain $i$ & $1 /$ time \\
$p_{i}$ & proportion of vertical transmission for strain $i$ & dimensionless \\
$H$ & (maximum) per-host predation rate & vectors/host/time \\
$\rho_{i}$ & proportion of hosts infected after consuming a vector & hosts/vector \\
& infected by strain $i$ & \\
\hline & &
\end{tabular}

hosts, that is, for high vector-host ratios $Q$. Also, it is important to notice that although hosts get infected from different transmission routes (stercorarian, oral and vertical), vectors can only get infected with the classical mode of transmission via bloodmeal.

\subsection{Model formulation}

To study the competition between two T. cruzi strains, we extend the 1-strain dynamical system of [13] used to model the transmission of sylvatic T. cruzi to a 2-strain system. We assume for simplicity that no coinfection occurs among hosts and vectors. This is most likely true for hosts [18], but not for vectors, in which mixed infections are common according to some studies [19]. Simple demographics, e.g., logistic reproduction and linear per capita mortality, are considered for both host and vector. Since we consider established populations, 
and host demographics are independent of host-vector contacts, we will assume the total host density $N_{h}$ to have reached its limiting value and write it without time dependence; some simple analysis of the equations below shows it to be asymptotically constant. We consider only female hosts, and assume that a proportion $p_{i}(i=1,2)$ of hosts infected with strain $i$ give birth to infected offspring. Variables and parameters are summarized in Table 1 and the resulting model is given by the following system of ordinary differential equations:

$$
\begin{aligned}
S_{h}^{\prime}(t)= & \left(1-\frac{p_{1} I_{h 1}(t)+p_{2} I_{h 2}(t)}{N_{h}}\right) g_{h}\left(N_{h}\right)-\left[c_{h 1}(Q(t))+\rho_{1} E_{h}(Q(t))\right] S_{h}(t) I_{v 1}(t) / N_{v}(t) \\
& -\left[c_{h 2}(Q(t))+\rho_{2} E_{h}(Q(t))\right] S_{h}(t) I_{v 2}(t) / N_{v}(t)-\mu_{h} S_{h}(t), \\
I_{h 1}^{\prime}(t)= & p_{1} \frac{I_{h 1}(t)}{N_{h}} g_{h}\left(N_{h}\right)+\left[c_{h 1}(Q(t))+\rho_{1} E_{h}(Q(t))\right] S_{h}(t) I_{v 1}(t) / N_{v}(t)-\mu_{h} I_{h 1}(t), \\
I_{h 2}^{\prime}(t)= & p_{2} \frac{I_{h 2}(t)}{N_{h}} g_{h}\left(N_{h}\right)+\left[c_{h 2}(Q(t))+\rho_{2} E_{h}(Q(t))\right] S_{h}(t) I_{v 2}(t) / N_{v}(t)-\mu_{h} I_{h 2}(t), \\
S_{v}^{\prime}(t)= & g_{v}\left(N_{v}(t)\right)-c_{v 1}(Q(t)) S_{v}(t) \frac{I_{h 1}(t)}{N_{h}}-c_{v 2}(Q(t)) S_{v}(t) \frac{I_{h 2}(t)}{N_{h}}-\mu_{v} S_{v}(t) \\
& -E_{h}(Q(t)) N_{h} \frac{S_{v}(t)}{N_{v}(t)}, \\
I_{v 1}^{\prime}(t)= & c_{v 1}(Q(t)) S_{v}(t) I_{h 1}(t) / N_{h}-\mu_{v} I_{v 1}(t)-E_{h}(Q(t)) N_{h} I_{v 1}(t) / N_{v}(t), \\
I_{v 2}^{\prime}(t)= & c_{v 2}(Q(t)) S_{v}(t) I_{h 2}(t) / N_{h}-\mu_{v} I_{v 2}(t)-E_{h}(Q(t)) N_{h} I_{v 2}(t) / N_{v}(t),
\end{aligned}
$$

where the total host density is $N_{h}=S_{h}+I_{h 1}+I_{h 2}$, the total host growth rate $g_{h}\left(N_{h}\right)=$ $r_{h} N_{h}\left(1-\frac{N_{h}}{K_{h}}\right)$ is assumed logistic, and similarly for the total vector density $N_{v}$ and total vector growth rate $g_{v}\left(N_{v}(t)\right)$.

\subsection{Analysis and reproductive numbers}

This model can be analyzed using standard techniques from dynamical systems theory focusing on equilibrium stability. Results are summarized here, with further details provided in references and appendix.

The first important result is the observation that, because by assumption disease incidence does not affect host or vector demographics (fecundity and mortality), the vector-host ratio $Q$ approaches a fixed value $Q^{*}$ regardless of the infection dynamics of either strain. The decoupling can be seen simply by summing the appropriate equations in (5) to write equations for the total host and vector densities:

$$
\begin{aligned}
& N_{h}^{\prime}(t)=g_{h}\left(N_{h}(t)\right)-\mu_{h} N_{h}(t), \\
& N_{v}^{\prime}(t)=g_{v}\left(N_{v}(t)\right)-\mu_{v} N_{v}(t)-E_{h}(Q(t)) N_{h}(t) .
\end{aligned}
$$

Analysis of these equations, detailed in $[11,12]$, shows that for most forms of the birth rate functions $g_{h}\left(N_{h}\right)$ and $g_{v}\left(N_{v}\right)$, including constant and logistic, as well as for a wide variety of saturation functions $E_{h}$ (besides Holling type I as in (1)), the total host and vector densities approach asymptotically stable equilibria $N_{h}^{*}$ and $N_{v}^{*}$ (the vector equilibrium may be zero under excessive predation, $H>\left(r_{v}-\mu_{v}\right) Q_{h}$, but no such high predation on vectors by any $T$. cruzi host has been documented, let alone vector extinction, so we henceforth take $N_{v}^{*}>0$ ). 
The analysis also shows that one can therefore pass to a simpler model in which $N_{v}(t)$ and $N_{h}(t)$ are taken as constants (their equilibrium values), thus making their quotient $Q$ fixed as well, so that

$$
c_{h i}(Q)=\beta_{h i} \min \left(Q^{*} / Q_{v}, 1\right), \quad c_{v i}(Q)=\beta_{v i} \min \left(Q_{v} / Q^{*}, 1\right), \quad E_{h}(Q)=H \min \left(Q^{*} / Q_{h}, 1\right)
$$

are all constants with respect to time (regardless of whether $Q^{*}$ is greater or less than $Q_{h}$ and $\left.Q_{v}\right)$.

The resulting model can be further simplified by eliminating $S_{h}$, defining it as $N_{h}^{*}-I_{h 1}-I_{h 2}$, and similarly for $S_{v}$, and by introducing the following notation: $\tilde{\beta}_{h i}=c_{h i}\left(Q^{*}\right)+\rho_{i} E_{h}\left(Q^{*}\right)$, $\tilde{\beta}_{v i}=c_{v i}\left(Q^{*}\right)(i=1,2), \tilde{\mu}_{v}=\mu_{v}+E_{h}\left(Q^{*}\right) / Q^{*}$ (all with units $1 /$ time). Then the system becomes:

$$
\begin{aligned}
& I_{h 1}^{\prime}(t)=p_{1} \mu_{h} I_{h 1}(t)+\tilde{\beta}_{h 1} S_{h}(t) I_{v 1}(t) / N_{v}-\mu_{h} I_{h 1}(t), \\
& I_{v 1}^{\prime}(t)=\tilde{\beta}_{v 1} S_{v}(t) I_{h 1}(t) / N_{h}-\tilde{\mu}_{v} I_{v 1}(t), \\
& I_{h 2}^{\prime}(t)=p_{2} \mu_{h} I_{h 2}(t)+\tilde{\beta}_{h 2} S_{h}(t) I_{v 2}(t) / N_{v}-\mu_{h} I_{h 2}(t), \\
& I_{v 2}^{\prime}(t)=\tilde{\beta}_{v 2} S_{v}(t) I_{h 2}(t) / N_{h}-\tilde{\mu}_{v} I_{v 2}(t),
\end{aligned}
$$

which can be shown to exhibit competitive exclusion, similar to other previously studied models for competing pathogen strains with cross-immunity [33, 34]. For further details see the analysis in [34], which deals with a similar model.

The outcome of this competition in the infection dynamics described by (8) is determined by certain well-defined epidemiological threshold quantities, the basic reproductive numbers $R_{1}$ and $R_{2}$ and the invasion reproductive numbers $\tilde{R}_{1}$ and $\tilde{R}_{2}$ for strains 1 and 2 , respectively. The basic reproductive number for a given strain gives the mean number of secondary infections produced by a single infected individual (host or vector) introduced into a completely susceptible population. The invasion reproductive number (IRN) gives instead the mean number of secondary infections of the given strain produced by a single infected individual introduced into a population in which the other strain is already endemic [35, 36]. In a cross-immunity context, therefore, $\tilde{R}_{i}<R_{i}$. Both reproductive numbers can be calculated using next-generation operator approaches [37, 38] (for the IRN, only the invading strain is considered to be an infection, and the endemic equilibrium for the resident strain is used in place of the disease-free equilibrium); the results are as follows, with details in the appendix:

$$
\begin{gathered}
R_{1}=\frac{1}{2}\left(p_{1}+\sqrt{p_{1}^{2}+4 \frac{\tilde{\beta}_{h 1} \tilde{\beta}_{v 1}}{\mu_{h} \tilde{\mu}_{v}}}\right), R_{2}=\frac{1}{2}\left(p_{2}+\sqrt{p_{2}^{2}+4 \frac{\tilde{\beta}_{h 2} \tilde{\beta}_{v 2}}{\mu_{h} \tilde{\mu}_{v}}}\right), \\
\tilde{R}_{1}=\frac{1}{2}\left(p_{1}+\sqrt{p_{1}^{2}+4\left(1-p_{2}\right) \frac{\tilde{\beta}_{h 1} \tilde{\beta}_{v 1}}{\tilde{\beta}_{h 2} \tilde{\beta}_{v 2}}}\right), \tilde{R}_{2}=\frac{1}{2}\left(p_{2}+\sqrt{p_{2}^{2}+4\left(1-p_{1}\right) \frac{\tilde{\beta}_{h 2} \tilde{\beta}_{v 2}}{\tilde{\beta}_{h 1} \tilde{\beta}_{v 1}}}\right) .
\end{gathered}
$$

A strain is able to invade (and persist in) a naive population when its basic reproductive number exceeds 1 ; in cases where both strains' basic reproductive numbers exceed 1, only a strain whose invasion reproductive number exceeds 1 can invade (and persist in) a population where the other strain is resident. The competitive exclusion comes from the fact that (as a little algebra shows) only one of the two IRNs can exceed 1:

$$
\tilde{R}_{1}>1 \Leftrightarrow \frac{\tilde{\beta}_{h 1} \tilde{\beta}_{v 1}}{\left(1-p_{1}\right)}>\frac{\tilde{\beta}_{h 2} \tilde{\beta}_{v 2}}{\left(1-p_{2}\right)} \Leftrightarrow \tilde{R}_{2}<1 .
$$


The IRN therefore permits one to derive a fitness measure for each strain. The dimensionless, reproductive-number-like quantity $\bar{M}_{i}=\tilde{\beta}_{h i} \tilde{\beta}_{v i} /\left[\left(1-p_{i}\right) \mu_{h} \tilde{\mu}_{v}\right]$ serves as such a measure, since (by the above inequalities) whichever strain has the higher value of $\bar{M}_{i}$ wins the competition.

\section{The role of the vector-host ratio in interstrain competition}

\subsection{Mathematical analysis}

The role that the vector-host ratio $Q$ plays in the competition between the two T. cruzi strains cocirculating in the SE US can be determined using the fitness measure derived through the model analysis in the preceding section. First, however, we simplify that measure by removing the portion of the $\bar{M}_{i}$ unrelated to $Q$, namely the mortality rates; we therefore define (using $Q$ instead of $Q^{*}$ to simplify notation):

$$
M_{i}(Q)=\frac{\left[c_{h i}(Q)+\rho_{i} E_{h}(Q)\right] c_{v i}(Q)}{1-p_{i}}=\frac{\left[\beta_{h i} \min \left(\frac{Q}{Q_{v}}, 1\right)+\rho_{i} H \min \left(\frac{Q}{Q_{h}}, 1\right)\right] \beta_{v i} \min \left(\frac{Q_{v}}{Q}, 1\right)}{1-p_{i}} .
$$

Thus, as before, $\tilde{R}_{1}>1 \Leftrightarrow M_{1}>M_{2} \Leftrightarrow \tilde{R}_{2}<1$, and whichever strain has a greater value of $M_{i}$ wins the competition (this actually holds for any form of $c_{h i}(Q), c_{v i}(Q), E_{h}(Q)$ as long as host and vector density dynamics remain decoupled from infection dynamics, so that $Q \rightarrow Q^{*}$ ).

Given the evidence available in the T. cruzi system zoonotic to the SE US, we consider that the two pathogen strains 1 and 2 utilize different strategies regarding the trade-off between vertical and horizontal transmissions. We therefore assume that $\beta_{h 1}>\beta_{h 2}$ and $p_{1}<p_{2}$. Because of the lack of precise data and to simplify the analysis we hypothesize that strains 1 and 2 do not differ regarding the efficiency of bloodmeal transmission, which is the only way to get infected vectors. Thus we take $\beta_{v 1}=\beta_{v 2}=\beta_{v}$. Finally, as regards the efficiency of oral transmission, we assume that strain 1 is not significantly better transmitted in this way than strain 2 (because oral transmission is likely to be the dominant infection pathway among raccoons [13], strain 2 which is generally associated with raccoons [15] may be better adapted to oral transmission than strain 1 , but in the absence of data to that effect we choose to make a considerably weaker assumption here). To simplify the analysis (see below), we assume that $\rho_{1}$ is less than $\frac{1-p_{1}}{1-p_{2}}$ times as great as $\rho_{2}$ (by assumption $\frac{1-p_{1}}{1-p_{2}}>1$ ), which includes the case $\rho_{1} \leq \rho_{2}$ - that is, strain 1 cannot have an advantage in oral transmission that is greater than strain 2's advantage from vertical transmission.

The vector-host ratio $Q$ may affect the competition of the two pathogen strains if the order of the $M_{i}$ (in particular, which of the two is larger) changes as a function of $Q$. We first show that the $M_{i}$ cannot cross when $Q$ is either smaller or larger than both $Q_{h}$ and $Q_{v}$ (regardless of whether $Q_{h}<Q_{v}$ or $\left.Q_{h}>Q_{v}\right)$. The values of $M_{i}$ in the different $\left(Q_{h}\right.$, $\left.Q_{v}\right)$ intervals are reported in Table 2 . The $M_{i}$ cannot cross on $\left(0, \min \left(Q_{h}, Q_{v}\right)\right]$ since they are both linear from the origin, nor on $\left[\max \left(Q_{h}, Q_{v}\right), \infty\right)$ since they are both multiples of the same hyperbola (unless in either case they are identical on the entire interval). When $Q_{h} \leq Q \leq Q_{v}, M_{i}$ exhibits a slower linear increase than on $\left(0, Q_{h}\right]$, and when $Q_{v} \leq Q \leq Q_{h}$, a hyperbolic decrease asymptotic to $\frac{\rho_{i} H \beta_{v}}{1-p_{i}} \frac{Q_{v}}{Q_{h}}$. In both cases, since the parts of $M_{i}(Q)$ which 

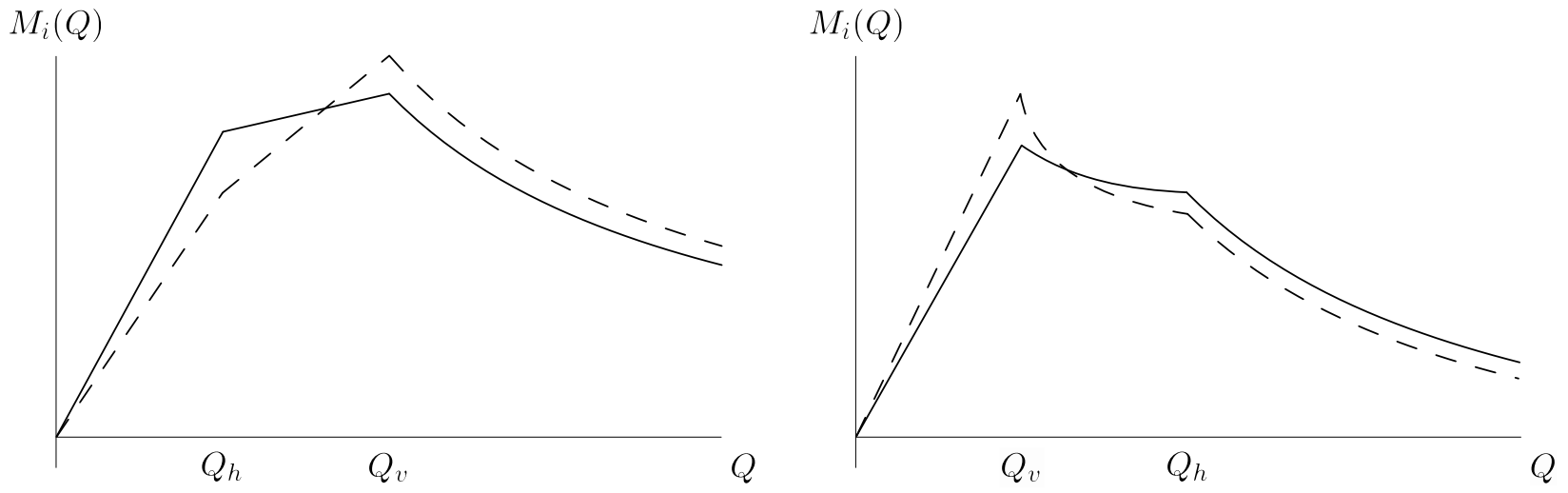

Figure 4: Fitness measures $M_{1}$ (dashed lines) and $M_{2}$ (solid lines) of respective strains as a function of $Q$. The only possibility for the two functions to intersect is between $Q_{h}$ and $Q_{v}$. When they do intersect, $M_{1}$ dominates for larger values of $Q$ if $Q_{h}<Q_{v}$ (left graph) and for smaller values of $Q$ if $Q_{h}>Q_{v}$ (right graph).

differ by strain are linear in Q (cf. Table 2), the $M_{i}$ can cross at most once between $Q_{h}$ and $Q_{v}$, unless they are identical for all $Q\left(\frac{\beta_{h 1}}{1-p_{1}}=\frac{\beta_{h 2}}{1-p_{2}}\right.$ and $\left.\frac{\rho_{1} H}{1-p_{1}}=\frac{\rho_{2} H}{1-p_{2}}\right)$. Thus, either $M_{i}$ dominates $M_{j}(i \neq j)$ for all values of $Q$, or else they cross somewhere in between $Q_{h}$ and $Q_{v}$. In cases where the $M_{i}$ cross between $Q_{h}$ and $Q_{v}$, one strain wins the competition for low vector-host ratios, say $Q<Q_{c}$ for some critical level $Q_{c}$, while the other strain wins for high vector-host ratios, $Q>Q_{c}$.

Table 2: Values of $M_{i}$ on the different $\left(Q_{h}, Q_{v}\right)$ intervals

\begin{tabular}{cll}
\hline$\left(0, \min \left(Q_{h}, Q_{v}\right)\right]$ & $\left(\min \left(Q_{h}, Q_{v}\right), \max \left(Q_{h}, Q_{v}\right)\right)$ & {$\left[\max \left(Q_{h}, Q_{v}\right), \infty\right)$} \\
\hline$\left[\frac{\beta_{h i}}{Q_{v}}+\frac{\rho_{i} H}{Q_{h}}\right] \frac{\beta_{v}}{1-p_{i}} \cdot Q$ & {$\left[\frac{\beta_{h i}}{Q_{v}} \cdot Q+\rho_{i} H\right] \frac{\beta_{v}}{1-p_{i}} \quad$ if $Q_{h}<Q_{v}$} & \\
& {$\left[\beta_{h i}+\rho_{i} H \frac{Q}{Q_{h}}\right] \frac{\beta_{v}}{1-p_{i}} \cdot \frac{Q_{v}}{Q} \quad$ if $Q_{v}<Q_{h}$} & {$\left[\beta_{h i}+\rho_{i} H\right] \frac{\beta_{v} Q_{v}}{1-p_{i}} \cdot \frac{1}{Q}$}
\end{tabular}

An investigation of the role of $Q$ in the outcome of the competition between the two strains therefore focuses on the $M_{i}$ values at the two saturation thresholds,

$M_{i}\left(Q_{h}\right)=\frac{\left[\beta_{h i} \min \left(\frac{Q_{h}}{Q_{v}}, 1\right)+\rho_{i} H\right] \beta_{v} \min \left(\frac{Q_{v}}{Q_{h}}, 1\right)}{1-p_{i}}$ and $M_{i}\left(Q_{v}\right)=\frac{\left[\beta_{h i}+\rho_{i} H \min \left(\frac{Q_{v}}{Q_{h}}, 1\right)\right] \beta_{v}}{1-p_{i}}$.

The only way for $Q$ to affect the competition is to have $M_{i}\left(Q_{h}\right)<M_{j}\left(Q_{h}\right)$ for some $i \neq j$ but $M_{i}\left(Q_{v}\right)>M_{j}\left(Q_{v}\right)$. If we solve both inequalities for $\beta_{h 1}$ we can show that $M_{1}\left(Q_{v}\right)>M_{2}\left(Q_{v}\right)$ 

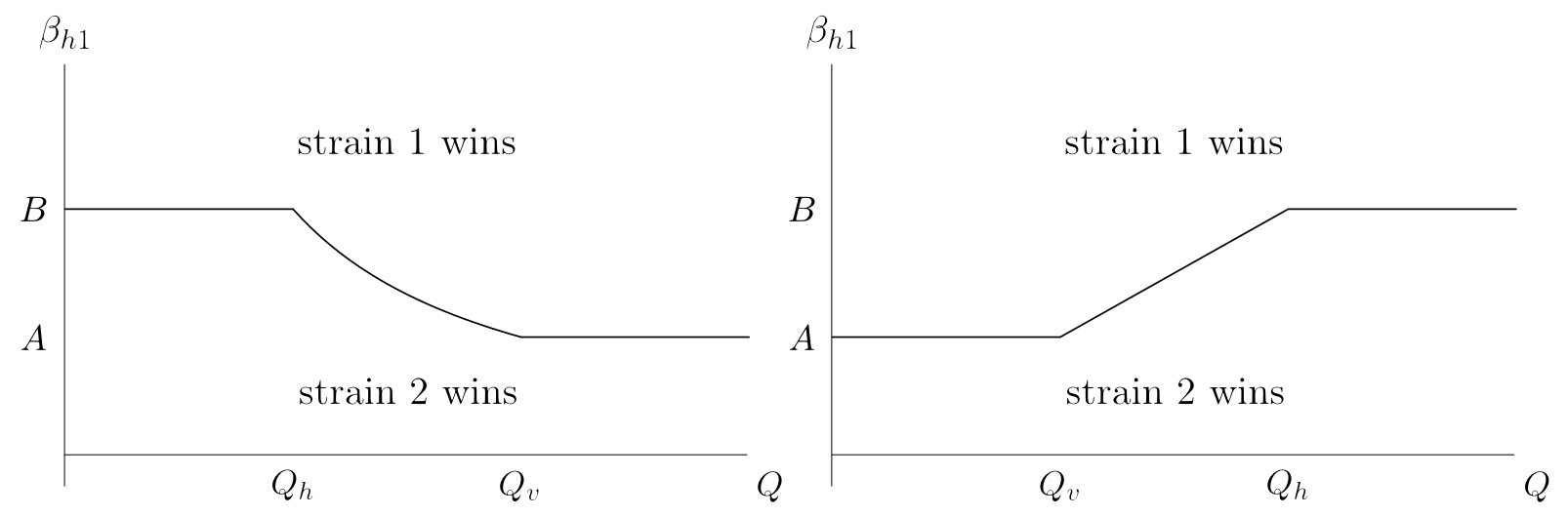

Figure 5: Competition outcomes as a function of $Q$ and $\beta_{h 1}$ for $Q_{h}<Q_{v}$ (left graph) and $Q_{h}>Q_{v}$ (right graph). The two limiting thresholds for $\beta_{h 1}$ are given in (9) and (10).

if and only if $\beta_{h 1}>A$, with

$$
A=\beta_{h 2} \frac{1-p_{1}}{1-p_{2}}+H \min \left(\frac{Q_{v}}{Q_{h}}, 1\right)\left[\rho_{2} \frac{1-p_{1}}{1-p_{2}}-\rho_{1}\right]
$$

and that $M_{1}\left(Q_{h}\right)>M_{2}\left(Q_{h}\right)$ if and only if $\beta_{h 1}>B$, with

$$
B=\beta_{h 2} \frac{1-p_{1}}{1-p_{2}}+H \max \left(\frac{Q_{v}}{Q_{h}}, 1\right)\left[\rho_{2} \frac{1-p_{1}}{1-p_{2}}-\rho_{1}\right] .
$$

Provided that the quantity in square brackets is positive (which it is by assumption on $\rho_{1}$, see above), then $A<B, \operatorname{since} \min \left(\frac{Q_{v}}{Q_{h}}, 1\right)<\max \left(\frac{Q_{v}}{Q_{h}}, 1\right)$ as long as $Q_{v} \neq Q_{h}$. Thus the condition for $Q$ to affect the outcome of the competition between the two strains is $A<\beta_{h 1}<B$, and consequently $M_{1}\left(Q_{h}\right)<M_{2}\left(Q_{h}\right)$ (since $\beta_{h 1}<B$ ) but $M_{1}\left(Q_{v}\right)>M_{2}\left(Q_{v}\right)$ (since $A<\beta_{h 1}$ ). These different possibilities (depending on the relative order of $Q_{h}$ and $Q_{v}$ ) are illustrated in Figure 4. In the case $Q_{h}<Q_{v}$, then strain 1 wins for $Q>Q_{c}$ while strain 2 wins for $Q<Q_{c}$ where

$$
\frac{Q_{c}}{Q_{v}}=\frac{\rho_{2} H\left(1-p_{1}\right)-\rho_{1} H\left(1-p_{2}\right)}{\beta_{h 1}\left(1-p_{2}\right)-\beta_{h 2}\left(1-p_{1}\right)} .
$$

In the case $Q_{h}>Q_{v}$, strain 1 wins for $Q<Q_{c}$ while strain 2 wins for $Q>Q_{c}$ where

$$
\frac{Q_{c}}{Q_{h}}=\frac{\beta_{h 1}\left(1-p_{2}\right)-\beta_{h 2}\left(1-p_{1}\right)}{\rho_{2} H\left(1-p_{1}\right)-\rho_{1} H\left(1-p_{2}\right)} .
$$

These results are depicted graphically in Figure 5.

We observe that this result holds even if there is no difference in oral transmissibility, $\rho_{1}=\rho_{2}$. However, if there is no oral transmission at all, $\rho_{1}=\rho_{2}=0$, or if the two contact processes saturate at the same threshold, $Q_{h}=Q_{v}$, the interval closes $(A=B)$, and the competition becomes independent of $Q$ (this is mathematically equivalent to removing the middle segment from the $M_{i}$ graphs). 
A similar analysis under the hypothesis that the two contact processes instead undergo Holling type II saturation, with $E_{h}(Q)=Q /\left(Q+Q_{h}\right)$ and similarly for $c_{h i}$ and (in $\left.1 / Q\right) c_{v i}$, produces identical results, down to the same expressions for $A$ and $B$. In this case one takes from

$$
M_{i}=\left(\beta_{h i} \frac{Q}{Q+Q_{v}}+\rho_{i} H \frac{Q}{Q+Q_{h}}\right) \frac{1}{1-p_{i}} \cdot \beta_{v} \frac{Q_{v}}{Q+Q_{v}}
$$

the portion which differs by strain,

$$
\hat{M}_{i}=\left(\beta_{h i} \frac{Q}{Q+Q_{v}}+\rho_{i} H \frac{Q}{Q+Q_{h}}\right) \frac{1}{1-p_{i}},
$$

and studies for which values of $Q \hat{M}_{1}(Q)>\hat{M}_{2}(Q)$. The only difference from the analysis given above is the expression for the critical value,

$$
Q_{c}=-\frac{\left(\beta_{h 1}-\beta_{h 2} \frac{1-p_{1}}{1-p_{2}}\right) Q_{h}-\left(\rho_{2} \frac{1-p_{1}}{1-p_{2}}-\rho_{1}\right) H Q_{v}}{\left(\beta_{h 1}-\beta_{h 2} \frac{1-p_{1}}{1-p_{2}}\right)-\left(\rho_{2} \frac{1-p_{1}}{1-p_{2}}-\rho_{1}\right) H} .
$$

\subsection{Fit with available data}

Whether the conditions for the vector-host ratio to affect the competition between the two $T$. cruzi strains can be satisfied and explain parasite specializations in terms of horizontal and vertical transmissions is an interesting question to ask. Data on the epidemiological traits of T. cruzi I and IIa which differ according to the geographical area of origin and the host considered and on vector and host densities are however crucially lacking, and the few laboratory estimates available probably fairly disconnected from field reality. Caution is therefore taken in this section in which we attempt to fit available data to the model.

If we use the data on the probability of vertical transmission in mice of [23], i.e., $p_{1}=1 / 3$ and $p_{2}=2 / 3$, and the estimates of the probability of oral transmission of [10], and assume that T. cruzi I and IIa are equally transmitted via this pathway, i.e., $\rho_{1}=\rho_{2}=0.177$ host/vector, the condition $A<\beta_{h 1}<B$ for $Q$ to affect the outcome of the competition between the two strains (see section 3.1) becomes: $2 \beta_{h 2}+0.177 H<\beta_{h 1}<2 \beta_{h 2}+0.177 H Q_{v} / Q_{h}$ when $Q_{h}<Q_{v}$ and $2 \beta_{h 2}+0.177 H Q_{v} / Q_{h}<\beta_{h 1}<2 \beta_{h 2}+0.177 H$ when $Q_{h}<Q_{v}$. Even if no precise data is available for the maximum rate at which hosts consume vectors in the field, $H$, the consumption of one vector per host and per year has been shown numerically to be sufficient to account for the observed T.cruzi prevalences in the SE US [13]. We interpret this estimate to reflect a low host preference for vectors as food (hence low $H$ ), which in turn makes it easier to satisfy this preference even when vectors are not abundant (i.e., low $\left.Q_{h}\right)$. Indeed, rough estimates of the threshold vector-host ratios have been made based on the rate at which vectors are spotted by humans for $Q_{h}$, which leads to a value $Q_{h}=10$ and on the maximum rate at which hosts can tolerate bites $b_{h}$ and the preferred biting rate of vectors $b_{v}$ for $Q_{v}$, which leads to a value of $Q_{v}=20$ for woodrats and $Q_{v}=400$ for raccoons and opossums (and henceforth, $Q_{h}<Q_{v}$ ) [13]. No data on the infection rates $\beta_{h}$ of the two strains are available, but if we take an example value of $\beta_{h 2}=0.5$ per year and the estimates mentioned above, this would bound $\beta_{h 1}$ between 1.177 and 1.254 per year for woodrats and 1.177 and 8.08 per year for raccoons and opossums. Even if the parameter values used here 
are rough estimations, one can see from this example that strain I has to be quite significantly better than strain IIa at stercorarian transmission for the condition to be respected, and that the range of possible values for strain I's stercorarian infection rate broadens as the difference between the two threshold vector-host ratios for saturation of the contact processes increases.

Whether the different host and vector species associations observed throughout the Americas, and in particular the ratio of their densities, can explain the evolution of T. cruzi and the observed specializations for one mode of transmission or another also needs to be examined. In the SE US, the vector-host ratio varies drastically depending on the transmission cycle considered. Estimations of population densities of hosts and vectors give rise to $Q$ values of 14 for woodrats, 1600 for raccoons and 3200 for opossums [10]. If we consider the threshold vectorhost ratios for saturation of the contact processes provided above, we obtain for woodrats a situation $Q_{h}<Q<Q_{v}$, in which the vector/host ratio can shift the advantage from one strain to the other. This is in accordance with the fact that both T. cruzi strains have been observed in woodrats in South Texas [39]. For raccoons and opossums, these estimations lead to $Q_{h}<Q_{v}<Q$. Our model therefore predicts that $T$. cruzi $\mathrm{I}$ is at maximum advantage among raccoons and opossums, and it is indeed prevalent among opossums (which happen to be immune to T. cruzi IIa [44], see discussion), but not among raccoons, which are mostly found infected with $T$. cruzi IIa in the SE US $[15,30]$. The superiority of $T$. cruzi I over IIa in stercorarian infectivity to raccoons may therefore not be great enough $\left(\beta_{h 1}<A\right)$ to capitalize on this advantage. Any discrepancy between model predictions and field observations may come from caveats in the measurement or estimation of the actual vector-host ratios. It could also suggest that conditions for the vector-host ratio to affect the competition between the two strains mentioned earlier are not met, or that other factors not considered in our model, in combination with the vector-host ratio, play a role in the evolution of the parasite.

\section{Discussion}

We analysed a two-strain epidemiological model describing the sylvatic transmission of Trypanosoma cruzi in a vector-host population with cross-immunity, considering saturation processes in the host-vector contact rates, and assuming that the two pathogen strains differ in their efficiencies at vertical and horizontal transmissions. We were able to show that for certain parameter combinations, the vector per host ratio affects the outcome of the competition between the two strains. This result holds providing that oral transmission was included in the model as a possible pathway for T. cruzi transmission, even if the two strains' efficiencies at this transmission mode do not differ, that oral and classical (stercorarian) transmissions saturated at different vector-host ratios, and occurs only between the two saturation thresholds (outside this interval, one of the two strains always wins the competition). Even if scattered data and rough estimates of parameters prevent any precise comparison of our model predictions with the biological reality in the field, they suggest that such conditions could reasonably be satisfied in some cases. This theoretical study opens new perspectives for understanding the specialization of $T$. cruzi for one transmission route or another. It also brings original and new elements to the comprehension of the evolution of alternative transmission modes in vector-borne diseases. 
This study is, to our knowledge, the first one to look at the evolution of the trade-off between vertical and horizontal transmissions independently of the evolution of pathogen virulence. Increased levels of virulence have been shown, both theoretically and experimentally, to favor horizontal transmission but to reduce the probability of vertical transmission, as virulence is generally negatively correlated to host fitness $[1,2,4]$. Thieme and collaborators $[40,41,42]$ studied a model for a directly-transmitted pathogen in which two competing strains were able to coexist in a single host population, but in addition to the direct transmission, the coexistence required that one strain be transmitted purely vertically and that both strains induce either a reduced fertility or additional mortality in hosts. In our model, we did not consider infected hosts to be submitted to an additive mortality rate due to infection compared to susceptible hosts, as is usually done in models of pathogen evolution. This assumption is reasonable given that parasite-induced mortality has never been reported among SE US T. cruzi sylvatic cycles. The T. cruzi strains present in the SE US are known to induce only mild symptoms to their most common hosts such as raccoons [17] or opossums [16]. Of course, because such hosts are wild animals, long-term impacts of infection in the field are hardly documented. Also, we do not have any precise data about differences in the epidemiological behaviours of the US T. cruzi strains. The T. cruzi I strains present in Latin America typically develop larger parasitemias in hosts and are more virulent than T. cruzi IIa in terms of parasite-induced mortality $[23,27]$. In Latin America, and possibly in the US if this holds true for the US strains, T. cruzi I higher virulence and efficiency at bloodmeal transmission (which is generally positively linked to parasitemia) could provide an additional advantage to this strain in terms of horizontal transmission and a disadvantage in terms of vertical transmission, and eventually change the predictions of our model. The addition of virulence would greatly complicate the analysis of the model but may prove that virulence and host and vector densities act together on the evolution of transmission modes. Our work nevertheless has the merit to demonstrate that evolution of transmission modes can decouple from evolution of virulence.

In interacting populations such as predator-prey or host-vector populations, the relative number of the two partners has a strong influence on the rate at which predation or disease transmission occurs $[10,31]$. The model of Choisy and collaborators investigates the evolutionary mechanisms behind parasite switching from simple life cycles to complex ones, the latter involving an intermediate host being a prey and a definitive host being a predator [5]. It shows that switching to a complex life cycle requires that the density of intermediate hosts is superior to the density of definitive hosts [5]. Our model predicts that high vector-host ratios select for vector-borne instead of vertical transmission in the case $Q_{h}<Q_{v}$, which accords with available estimates of vector-host ratios in the SE US T. cruzi system (see section 3.2). Even if these estimates must be taken with care, and that different selective processes are likely to occur in the two systems compared, Choisy et al. results strengthen our conclusion that relative densities of hosts play a key role in the evolution of transmission modes of multihost parasites.

To accurately test the role of vector-host ratio on the evolution of transmission modes of a vector-borne disease, we considered saturation processes in host-vector contacts and all possible relative densities of host and vectors. Indeed, compared to classical vector-borne 
disease models that assume that hosts are non-limiting and that are therefore only valid for low vector-host ratios $[8,9]$, we considered here (following $[10,11,12,13]$ ) that hosts can be limiting as well, which should reasonably occur in Chagas' disease as hosts typically become irritable when vectors are too numerous [14]. This enabled us to find conditions under which the two parasite strains are differentially advantaged along the range of possible vector-host ratios. In vector-borne diseases, two different types of contacts take place, either between an infected vector and a healthy host or between a healthy vector and an infected host. The infection rate of hosts is maximal for high $Q$ values, i.e., when vector density is relatively high compared to host density, and the infection rate of vectors maximal for low $Q$ values (see Figures 2 and 3). As a consequence, a vector-borne disease using the traditional route of transmission maximizes its infection rate, and consequently its prevalence, at intermediate $Q$ values, that is for $Q=Q_{v}$. This can be seen in Figure 4: in cases where the vector-host ratio matters, the pathogen better transmitted by stercorarian transmission route, T. cruzi I, always dominates at $Q=Q_{v}$, regardless of whether $Q_{h}<Q_{v}$ or $Q_{h}>Q_{v}$. Saturation processes have rarely been used in epidemiology to model vector-borne diseases (but see $[10,11,12,13]$ ). Here we show that they can nonetheless have a strong influence on the evolution of vectorborne diseases, as a combination of saturation processes in oral and horizontal transmissions is necessary for the vector-host ratio to influence the evolution of the trade-off between vertical and horizontal transmissions.

Surprisingly, oral transmission seems to play an important role in the outcome of the competition between the two strains, even if the efficiency of the two strains at being transmitted via this route is similar. Without a source of infections coming from the ingestion of infected vectors by susceptible hosts, or when the saturation thresholds of transmission by predation and by bloodmeals are the same, one of the two strains wins for all vector-host ratios. It is interesting to notice that vector consumption by hosts alone (i.e., $E_{h}(Q)>0$ ), without any possibility of transmission of the parasite via this route (i.e., $\rho_{1}=\rho_{2}=0$ ), is not sufficient to maintain the influence of $Q$ alone on the outcome of the competition between the two strains. This independence between the vector-host ratio and the evolution of transmission mode unless hosts dynamically consume infected vectors is consistent with the theoretical results of [43]. Morozov and Adamson showed that the evolution of virulence in a directly-transmitted pathogen is independent of host biodemographic parameters (e.g. carrying capacity, host growth rate), unless a predator dynamically consuming infected hosts is added to the system [43]. Whether host predation on vectors in general modifies the influence of host and vector biodemographic parameters on the evolution of pathogen virulence and/or transmission mode in a host-vector cycle warrants further study.

We simplified our model by considering a single host population. However, among sylvatic hosts in the US, T. cruzi I is generally associated with Virginia opossums and T. cruzi IIa with raccoons and other placental mammals [15]. In addition, opossums are immune to T. cruzi IIa [44]. The impossibility of vertical transmission in a marsupial host most certainly constitutes an important selective force affecting the specialisation of T. cruzi I toward horizontal and/or oral transmission modes. Further work, already in progress, extends the context of the present study to that of multiple interconnected transmission cycles by taking into account two host populations (with a common vector population) differentially infected by two pathogen strains. Also, we did not consider the possibility that mixed infections take 
place in vectors [19] and the fact that T. cruzi I might perform better in mixed infections, i.e., be better transmitted to hosts, compared to T. cruzi IIa [29]. We think that the main conclusion of our study would have been unchanged if we did, but that situations in which both strains can coexist would have arisen. However, the simpler model used in the present study is sufficient to illustrate the role of the vector-host ratio in the evolution of transmission modes from a broader perspective than T. cruzi transmission in the SE US.

In conclusion, the existence of multiple transmission routes, most driven by contact processes with different saturation thresholds, enables population dynamics - here measured by the vector-host ratio - to influence the evolution of the pathogen. Adaptation to a given route provides a competitive advantage at vector-host ratios where that route is most effective. In the case of $T$. cruzi, stercorarian and oral transmission to hosts stem from contact processes initiated by different populations (vectors and hosts, respectively), each therefore staking out a different interval of vector-host ratio values, while vertical transmission sidesteps this ratio entirely. This original approach which highlights multiple transmission routes and saturation processes in a vector-borne disease setting brings exciting new elements to the understanding of the evolution of pathogens in general, and of their transmission modes in particular. It promises further insights into the evolution of other pathogens.

Acknowledgment: This research was supported by the National Science Foundation under Grant DMS-1020880. We are very grateful to two anonymous reviewers who greatly helped us to improve the manuscript.

\section{Appendix. Model analysis}

This brief section provides additional computational details for the qualitative analysis of the infection dynamics. Examination of the steady-state conditions of system (8) yields three equilibria: the disease-free equilibrium $E_{0}(0,0,0,0)$, an equilibrium in which only strain 1 is endemic $E_{1}\left(x_{h 1}^{*} N_{h}, 0, x_{v 1}^{*} N_{v}, 0\right)$, and an equilibrium in which only strain 2 is endemic, $E_{2}\left(0, x_{h 2}^{*} N_{h}, 0, x_{v 2}^{*} N_{v}\right)$ (no coexistence endemic equilibrium exists). In the latter two cases, the equilibrium prevalence levels for the endemic strain $i$ are given by

$$
x_{h i}^{*}=\frac{\tilde{\beta}_{h i} \tilde{\beta}_{v i}-\left(1-p_{i}\right) \mu_{h} \tilde{\mu}_{v}}{\tilde{\beta}_{v i}\left(\tilde{\beta}_{h i}+\left(1-p_{i}\right) \mu_{h}\right)}, \quad x_{v i}^{*}=\frac{\tilde{\beta}_{h i} \tilde{\beta}_{v i}-\left(1-p_{i}\right) \mu_{h} \tilde{\mu}_{v}}{\tilde{\beta}_{h i}\left(\tilde{\beta}_{v i}+\tilde{\mu}_{v}\right)} .
$$

Existence and stability conditions for these equilibria, and thus the outcome of the competition between the two strains, are determined by the basic reproductive numbers $R_{1}$ and $R_{2}$ and the invasion reproductive numbers $\tilde{R}_{1}$ and $\tilde{R}_{2}$ for strains 1 and 2 . The standard next-generation operator approaches for calculating reproductive numbers $[37,38]$ involve decomposing reduced Jacobian matrices for the system (including only rows and columns corresponding to infective populations) as the difference of two matrices, the former a nonnegative matrix representing new infections only, and then computing the next-generation matrix as the product of the first matrix and the inverse of the second. For system (8), this 
computation is

$$
\begin{gathered}
{\left[\begin{array}{cccc}
p_{1} \mu_{h} & \tilde{\beta}_{h 1} / Q^{*} & 0 & 0 \\
\tilde{\beta}_{v 1} Q^{*} & 0 & 0 & 0 \\
0 & 0 & p_{2} \mu_{h} & \tilde{\beta}_{h 2} / Q^{*} \\
0 & 0 & \tilde{\beta}_{v 2} Q^{*} & 0
\end{array}\right] \cdot\left[\begin{array}{cccc}
\mu_{h} & 0 & 0 & 0 \\
0 & \mu_{v} & 0 & 0 \\
0 & 0 & \mu_{h} & 0 \\
0 & 0 & 0 & \mu_{v}
\end{array}\right]^{-1}} \\
\quad=\left[\begin{array}{cccc}
p_{1} & \tilde{\beta}_{h 1} / Q^{*} \mu_{v} & 0 & 0 \\
\tilde{\beta}_{v 1} Q^{*} / \mu_{h} & 0 & 0 & 0 \\
0 & 0 & p_{2} & \tilde{\beta}_{h 2} / Q^{*} \mu_{v} \\
0 & 0 & \tilde{\beta}_{v 2} Q^{*} / \mu_{h} & 0
\end{array}\right] .
\end{gathered}
$$

The reproductive number in question is then the dominant eigenvalue of the next-generation matrix. The derivations of these reproductive numbers establish local asymptotic stability of the three equilibria.

\section{References}

[1] R.M. Anderson, R.M. May, Coevolution of hosts and parasites, Parasitology 85 (1982) 411-426.

[2] P.E. Turner, V.S. Cooper, R.E. Lenski, Tradeoff between horizontal and vertical modes of transmission in bacterial plasmids, Evolution 52 (1998) 315-329.

[3] J.C. de Roode, A.J. Yates, S. Altizer, Virulence-transmission trade-offs and population divergence in virulence in a naturally occurring butterfly parasite, Proc. Natl Acad. Sci. U S A 105 (2008) 7489-7494.

[4] L. Lambrechts, T.W. Scott, Mode of transmission and the evolution of arbovirus virulence in mosquito vectors, Proc. R. Soc. B 276 (2009) 1369-1378.

[5] M. Choisy, S.P. Brown, K.D. Lafferty, F. Thomas, Evolution of trophic transmission in parasites: why add intermediate hosts? Am. Nat. 162 (2003) 172-181.

[6] M. Desai, F.O. ter Kuile, F. Nosten, R. McGready, K. Asamoa, B. Brabin, R.D. Newman, Epidemiology and burden of malaria in pregnancy, Lancet Infect. Dis. 7 (2007) 93-104.

[7] U. Kemmerling, C. Bosco, N. Galanti, Infection and invasion mechanisms of Trypanosoma cruzi in the congenital transmission of Chagas' disease: A proposal, Biol. Res. 43 (2010) $307-316$.

[8] R. Ross, The prevention of Malaria, second ed., Murray, London, 1911.

[9] J.X. Velasco-Hernández, A model for Chagas disease involving transmission by vectors and blood transfusion, Theor. Popul. Biol. 46 (1994) 1-31.

[10] C.M. Kribs-Zaleta, Estimating contact process saturation in sylvatic transmission of Trypanosoma cruzi in the United States, PloS Negl. Trop. Dis. 4 (2010) e656. 
[11] C.M. Kribs-Zaleta, Vector consumption and contact process saturation in sylvatic transmission of T. cruzi, Math. Popul. Stud. 13 (2006) 135-152.

[12] C.M. Kribs-Zaleta, Sharpness of saturation in harvesting and predation, Math. Biosci. Eng. 6 (2009) 719-742.

[13] C.M. Kribs-Zaleta, Alternative transmission modes for Trypanosoma cruzi, Math. Biosci. Eng. 7 (2010) 661-676.

[14] J.E. Rabinovich, P. Himschoot, A population-dynamics simulation model of the main vectors of Chagas' disease transmission, Rhodnius prolixus and Triatoma infestans, Ecol. Modell. 52 (1990) 249-266.

[15] D.M. Roellig, E.L. Brown, C. Barnabé, M. Tibayrenc, F.J. Steurer, M.J. Yabsley, Molecular typing of Trypanosoma cruzi isolates, United States, Emerg. Infect. Dis. 14 (2008) $1123-1125$.

[16] S.C. Barr, C.C. Brown, V.A. Dennis, T.R. Klei, The lesions and prevalence of Trypanosoma cruzi in opossums and armadillos from southern Louisiana, J. Parasitol. 77 (1991) 624-627.

[17] S.M. Pietrzak, O.J. Pung, Trypanosomiasis in raccoons from Georgia, J. Wildl. Dis. 34 (1998) 132-136.

[18] L. Norman, I.G. Kagan, Immunologic studies on Trypanosoma cruzi: II. Acquired immunity in mice infected with avirulent American strains of T. cruzi, J. Infect. Dis. 107 (1960) 168-174.

[19] M. Yeo, M.D. Lewis, H.J. Carrasco, N. Acosta, M. Llewellyn, S.A. da Silva Valente, V. de Costa Valente, A.R. de Arias, M.A. Miles, Resolution of multiclonal infections of Trypanosoma cruzi from naturally infected triatomine bugs and from experimentally infected mice by direct plating on a sensitive solid medium, Int. J. Parasitol. 37 (2007) 111-120.

[20] S.A. Kjos, K.F. Snowden, J.K. Olson, Biogeography and Trypanosoma cruzi infection prevalence of Chagas disease vectors in Texas, USA, Vector Borne Zoonotic Dis. 9 (2009) $41-49$.

[21] W.F. Pippin, The biology and vector capability of Triatoma sanguisuga texana Usinger and Triatoma gerstaeckeri compared with Rhodnius prolixus, J. Med. Entomol. 7 (1970) $30-45$.

[22] E.A. Moreno, I.M. Rivera, S.C. Moreno, M.E. Alarcón, A. Lugo-Yarbuh, Transmisión vertical de Trypanosoma cruzi en ratas Wistar durante la fase aguda de la infección (Vertical transmission of Trypanosoma cruzi in Wistar rats during the acute phase of infection), Invest. Clin. 44 (2003) 241-254.

[23] C.A. Hall, E.M. Pierce, A.N. Wimsatt, T. Hobby-Dolbeer, J. Bradley Meers, Virulence and vertical transmission of two genotypically and geographically diverse isolates of Trypanosoma cruzi in mice, J. Parasitol. 96 (2010) 371-376. 
[24] O. Sánchez Negrete, M.C. Mora, M.A. Basombrío, High prevalence of congenital Trypanosoma cruzi infection and family clustering in Salta, Argentina, Pediatrics 115 (2005) e668-e672.

[25] D.M. Roellig, A.E. Ellis, M.J. Yabsley, Oral transmission of Trypanosoma cruzi with opposing evidence for the theory of carnivory, J. Parasitol. 95 (2009) 360-364.

[26] J.E. Rabinovich, N. Schweigmann, V. Yohai, C. Wisnivesky-Colli, Probability of "Trypanosoma cruzi" transmission by Triatoma infestans (Hemiptera: Reduviidae) to the opossum Didelphis albiventris (Marsupialia: Didelphidae), Am. J. Trop. Med. Hyg. 65 (2001) 125-130.

[27] M.J. Yabsley, G.P. Noblet, Biological and molecular characterization of a raccoon isolate of Trypanosoma cruzi from South Carolina, J. Parasitol. 88 (2002) 1273-1276.

[28] M.G. Risso, G.B. Garbarino, E. Mocetti, O. Campetella, S.M. González Cappa, C.A. Buscaglia, M.S. Leguizamón, Differential expression of a virulence factor, the transsialidase, by the main Trypanosoma cruzi phylogenetic lineages, J. Infect. Dis. 189 (2004) $2250-2259$.

[29] C.A.C. Araújo, P.H. Cabello, A.M. Jansen, Growth behaviour of two Trypanosoma cruzi strains in single and mixed infections: in vitro and in the intestinal tract of the bloodsucking bug, Triatoma brasiliensis, Acta Trop. 101 (2007) 225-231.

[30] C.A. Hall, C. Polizzi, M.J. Yabsley, T.M. Norton, Trypanosoma cruzi prevalence and epidemiologic trends in lemurs on St. Catherine's Island, Georgia, J. Parasitol. 93 (2007) 93-96.

[31] R. Arditi, L.R. Ginzburg, Coupling in predator-prey dynamics: ratio-dependence, J. Theor. Biol. 139 (1989) 311-326.

[32] C.M. Kribs-Zaleta, To switch or taper off: the dynamics of saturation, Math. Biosci. 192 (2004) 137-152.

[33] H.J. Bremermann, H.R. Thieme, A competitive exclusion principle for pathogen virulence, J. Math. Biol. 27 (1989) 179-190.

[34] Z. Feng, J.X. Velasco-Hernández, Competitive exclusion in a vector-host model for the dengue fever, J. Math. Biol. 35 (1997) 523-544.

[35] T.C. Porco, S.M. Blower, Designing HIV vaccination policies: subtypes and crossimmunity, Interfaces 283 (1998) 167-190.

[36] P. Zhang, G.J. Sandland, Z. Feng, D. Xu, D.J. Minchella, Evolutionary implications for interactions between multiple strains of host and parasite, J. Theor. Biol. 248 (2007) $225-240$.

[37] O. Diekmann, J.A.P. Heersterbeek, J.A.J. Metz, On the definition and the computation of the basic reproduction ratio $R_{0}$ in models for infectious diseases in heterogeneous population, J. Math. Biol. 28 (1990) 365-382. 
[38] P. Van den Driessche, J. Watmough, Reproduction numbers and sub-threshold endemic equilibria for compartmental models of disease transmission, Math. Biosci. 180 (2002) $29-48$.

[39] R.A. Charles, S. Kjos, A.E. Ellis, J.C. Barnes, M.J. Yabsley, Southern plains woodrats (Neotoma micropus) from southern Texas are important reservoirs of two genotypes of Trypanosoma cruzi and host of a putative novel Trypanosoma species, Vector Borne Zoonotic Dis. (2012), in press.

[40] S.H. Faeth, K.P. Hadeler, H.R. Thieme, An apparent paradox of horizontal and vertical disease transmission, J. Biol. Dyn. 1(2007) 45-62.

[41] T. Dhirasakdanon, H.R. Thieme, Persistence of vertically transmitted parasite strains which protect against more virulent horizontally transmitted strains, in Z. Ma, Y. Zhou, J. Wu (Eds.), Modeling and dynamics of infectious diseases, World Scientific, 2009, pp. $187-215$.

[42] T. Dhirasakdanon, H.R. Thieme, Stability of the endemic coexistence equilibrium for one host and two parasites, Math. Model. Nat. Phenom. 5 (2010) 109-138.

[43] A.Y. Morozov, M.W Adamson, Evolution of virulence driven by predator-prey interaction: possible consequences for population dynamics, J. Theor. Biol. 276 (2011) 181-191.

[44] D.M. Roellig, A.E. Ellis, M.J. Yabsley, Genetically different isolates of Trypanosoma cruzi elicit different infection dynamics in raccoons (Procyon lotor) and Virginia opossums (Didelphis virginiana), Int. J. Parasitol. 39 (2009) 1603-1610. 